\title{
Review Paper: Emergency Medical Technicians' Ethical Challenges in the Prehospital Emergency Services: A Review Article
}

\author{
Fatemeh Cheraghi $^{1}$ (D, Mohammad Reza Yousefzadeh Chosari² (D, Mehdi Beyrami Jam³ (D, Ali Afshari ${ }^{* *}$ (D)
}

1. Chronic Disease (Home Care) Research center, School of Nursing \& Midwifery, Hamadan university of Medical Sciences, Hamedan, Iran

2. Department of Educational Sciences, Bu-Ali Sina University, Hamadan, Iran

3. Health in Emergency and Disaster Research Center, University of Social Welfare and Rehabilitation Sciences, Tehran, Iran

4. School of Nursing and Midwifery, Hamadan University of Medical Sciences, Hamadan, Iran.

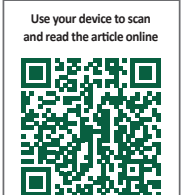

Citat on: Cheraghi F, Yousefzadeh Chosari MR, Beyrami Jam M, Afshari A. Emergency Medical Technicians' Ethical Challenges in the Prehospital Emergency Services: A Review Article. Health in Emergencies and Disasters Quarterly. 2019; 5(1):5-12. http:// dx.doi.org/10.32598/hdq.5.1.151.2

http://dx.doi.org/10.32598/hdq.5.1.151.2

\section{(c) (i) (8)}

Article info:

Received: 12 Jan 2019

Accepted: 25 Jul 2019

Available Online: 01 Oct 2019

\section{Keywords:}

Ethical challenges, Emergency

Medical Technicians (EMTs),

Prehospital emergency

\section{A B S T RACT}

Background: Because of the complexity, stressfulness, and the threatening nature of the working environment of prehospital Emergency Medical Technicians (EMTs), they encounter many ethical challenges during making decisions and providing quick and accurate care to patients. The present study aimed to investigate the ethical challenges among prehospital EMTs

Materials and Methods: To review all published studies, we searched SID, Medlib, Iran Medex, Magiran, Web of Science, Medline, Google Scholar, CINAHL, PubMed, Ovid, and Scopus databases from 2000-18. The keywords were "ethical challenges", "emergency medical technicians", and "prehospital emergency".

Results: According to the evaluation of the studies, the ethical challenge regarding prehospital emergencies included decision over dispatching an ambulance to the scene, cardiopulmonary resuscitation, triage in crisis and disaster, irrational interventions of the patient or patient's family, intervention in accordance with patient's informed consent, and safe driving.

Conclusion: Considering the unique conditions of prehospital caring, identifying, and discovering the ethical conflicts in this field of the health care system and providing the appropriate solution are very important. 


\section{Introduction}

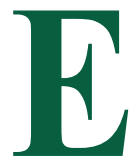

thical issues have become increasingly popular in recent years. In this regard, ethics was introduced as a research topic since 1970 [1]. Ethics is a set of values and rules that define right and wrong behavior and represent acceptable and unacceptable behaviors [2].

Ethics is considered as the study of behavioral standards and moral judgment that includes the four principles of usefulness, harmlessness, respect for independence, and justice. Health care personnel in each of these areas face different ethical challenges and conflicts [3]

Medical care is based on many interactions and events between healthcare providers and patients. In this process, many value choices, including ethical issues, can intrinsically and potentially be made based on the personal beliefs, commitment, responsibility, and habits of healthcare providers [4]. In some cases, however, patients and healthcare providers may disagree on certain values, which may cause ethical issues [5]. All healthcare providers face ethical challenges when caring for those in need, regardless of the context of care [6].

The services of the Emergency Medical System (EMS) are an essential part of the hospital and healthcare system [7]. Prehospital Emergency Medical Technicians (EMTs) are the first responders in emergencies, from severe traffic accidents, natural disasters, to minor injuries and illnesses. They experience various physical and emotional stresses to stabilize the patients' condition, to calm the patients and their anxious relatives, and to ensure the continuity of care until the patient's admission to treatment centers $[8,9]$.

All health care personnel, including EMTs, face ethical conflicts when providing care to patients [10]. Prehospital care is unique care characterized by factors that may lead to several ethical challenges compared to other care services in different situations [11]. To achieve an effective system in which personnel can make sound ethical decisions, they have to identify ethical challenges and conflicts [12].

Regarding the critical position of prehospital EMTs and the specific nature of the prehospital environment, we can conclude that the EMTs face numerous ethical issues while performing prehospital care. Therefore, it is necessary to identify these issues and probable solutions to prevent their consequences $[7,13]$. In this article, we considered the ethical dilemmas and challenges that might appear to the prehospital EMTs.

\section{Study Procedure}

We searched SID, Medlib, Iran Medex, Magiran, Medline, Web of Science, Google Scholar, CINAHL, SCOPUS, Ovid, and PubMed databases to review all published studies between 2000-18 with the keywords of "ethics", "ethical challenges", "ethical conflicts", "emergency medical technicians", "medical emergency", and "prehospital emergency". Then we selected and reviewed the articles with the subject matter of ethics in the field of medical emergencies.

After reviewing the titles and abstracts of the 212 selected articles in the prehospital field, 122 unrelated and of poor quality articles were excluded. Then 41 more articles were excluded because they were not available in full text and were written in different languages. Of 49 remaining articles, 12 foreign and 4 Iranian articles specifically addressed the ethical challenges of EMTs. We extracted challenges raised in these articles and presented them.

\section{Results}

In this study, we reviewed 16 articles related to ethics in prehospital activities. These articles comprised six crosssectional descriptive articles, four review articles, three qualitative articles, two observational study articles, and one case study. After reviewing and analyzing the results of the extracted articles, the ethical challenges of EMTs were categorized in eight main areas: whether to send an ambulance to the scene of the accident, cardiopulmonary resuscitation, triage in critical events and disasters, misconceptions and stigmas of patients, irrational patient/ patient's family interventions, informed consent, professional communication, and safe driving.

\section{Whether to send an ambulance to the scene of the accident}

Due to a lack of equipment, many EMS try to limit the dispatch of operational personnel and ambulances. Still, this procedure is not possible, since the staff of EMS are unaware of the situation of the scene and do not have a clear description of dispatch cases [14]. Emergency standards refer to what occurs to people (without interventions and treatments) that may harm their health or worsen their injury [15]. Since it is not always possible to provide a clear definition of an emergency, recognizing the need to dispatch or not an ambulance is a serious ethical issue in the EMS. 
In many cases, dispatching ambulances to non-emergency missions has deprived some emergency patients of access to the ambulance due to lack of or busy ambulances. Although some systems use telephone triage protocols to detect these cases [16], studies on the use of these protocols by medical emergency center operators have not been so recommended $[17,18]$.

\section{Cardiopulmonary Resuscitation}

Out-of-hospital cardiopulmonary arrest is one of the life-threatening conditions that can be controlled if the prehospital emergency care is taken. EMTs can save lives by performing prompt and timely cardiopulmonary resuscitation operations as well as using defibrillation [19]. The public expects prehospital service providers to initiate resuscitation at the time of cardiac arrest and transfer the patient to the health center in any circumstances [20]. However, factors such as guidelines and protocols for resuscitation operations, the safety and security of the rescue team, the conditions associated with legal considerations, and the cultural beliefs of the patient's family and companions may influence this action. These factors lead to a series of decisions about whether performing resuscitation, when to resume resuscitation, and whether to transfer the patient after unsuccessful resuscitation [21].

Despite a large number of out-of-hospital cardiac arrest cases, EMS only responds to $25 \%-50 \%$ of these cases [22, 23]. On the other hand, the survival of these patients depends on many factors, such as the primary cause of cardiac arrest, the age of the patient, the original disease, and the traumatic or non-traumatic status of the patient. Most cardiac arrest patients have no chance of survival outside the hospital, so developing protocols for stopping resuscitation at the scene of the incident is essential [24, 25].

The American Heart Association and the American Board of Surgeons and Trauma have stated some non-resuscitation conditions: 1 . uncertainty about the safety and security of the accident scene; 2 . clear signs of death such as decapitation, bruising dependent lividity, decomposition, and rigor mortis; 3 . clear evidence that the patient has not been resuscitated by a physician $[23,26]$. In 2000, it was recommended to suspend operations in prehospital conditions and when advanced cardiopulmonary resuscitation was unsuccessful after 20-30 minutes [27].

Also, in 2009, a guideline was issued stating that resuscitation should be stopped under the following conditions: the absence of reversible rhythm before transferring the patient, no sign of cardiac arrest, inadequate rhythm for shock, and no evidence of shock in the pa- tient [28]. Thus, considering these conditions and continuing to resuscitate the patient, the phrase "futile and potentially inappropriate interventions" was introduced for these patients $[29,30]$.

Most experts argue that despite resource constraints, it is incorrect to limit health care resources based on social costs [31]. Organ donation is another potential social reason for continuing apparently ineffective treatments. This means that efforts to continue resuscitation may be of more value for other people [32]. Given the limited supply of organs, it is advisable to take every opportunity to use the organs of patients who have had brain death but a normal cardiac function. Therefore, despite the ethical issues involved in "futile and potentially inappropriate interventions", these measures may lead to greater acceptance of prehospital services for the benefit of organ donation cases. However, given the complexity of this issue, EMS should develop evidence-based guidelines and protocols for such decisions. These protocols help clarify situations where complex ethical issues are involved so that prehospital service providers can make the best decision in cases such as cardiac arrest.

\section{Triage in critical events and disasters}

Moral challenges exist in the bedrock of various decisions during a crisis. In critical situations, because of excessive demand for available resources, organization managers, and system operating personnel face ethical challenges when deciding on the best way to allocate and distribute resources [33].

In critical situations, priorities in resource allocation shift from patient-centered to population-centered status. Also, during natural disasters, agents of the EMS seek to make use the most of the available resources so that more people can access the best resources and actions [34].

Under normal circumstances, the average survival rate for people with cases of cardiac arrest occurring outside of a hospital setting is approximately $3.3 \%$, and this figure is further reduced in critical moments due to time spent responding to large volumes of requests and actions [35]. As a result and to avoid spending significant amounts of resources on those with the least chance of survival during the crisis, EMS may face ethical issues in deciding whether to work to rescue patients in critical condition or not [34]. 


\section{Misconceptions and stigmas}

This situation will appear regarding the type of illness, the patient's position, and socio-cultural status [36]. Stigma will usually take place due to misconceptions of the technicians towards the patients before the initial interventions. For example, in dealing with patients with alcoholism, drug abuse, sexual abuse, or rape, the situation usually leads to have a negative picture of these patients, to reduce the delivered quality of care, and even in some cases, not to do the least medical care [37].

\section{Irrational patient/ patient's family inter- ventions}

One of the unreasonable actions of the patients or their families is the refusal to do treatment for the patient. Two ethical challenges raised are as follows: 1. the usefulness of treatment measures taken by EMS technicians, and 2. respect for patient's independence [38]. The critical point is, in these situations, whether the patient's decision-making power and confidence can be trusted. Second, can we examine the patient's decision-making power in this short time? However, the suggested guidelines may help in these issues, but it should be noted that the proposed protocols and guidelines in many countries are not clearly defined [7].

Another problem regarding irrational interventions of the patient or family and relatives is to refuse to transfer the patient to the health center. An EMT recognizes an emergency situation and considers patient in need of continued treatment in the hospital. Still, their relatives do not intend to transfer him/her to the medical center. Despite the explanations of the EMT and informing the complications and consequences of this refusal to transfer, the patient does not consent to be transferred to the hospital. In these cases, there will be a conflict between the duties of the staff and the patient's willingness [39].

\section{Informed consent}

Consent is one of the most common ethical challenges and conflicts in EMS. This is related to the situations in which the patient decides about his or her health [40]. But in most prehospital emergency cases, the patient is not in a position to make the right decision. The prehospital emergency system is an exception to intra-hospital guidelines for informed consent and does not require informed consent from the patient or their family for emergency action [41].

\section{Professional communications}

Working in the prehospital emergency requires cooperation, correspondence, and assistance of technicians [42]. When disagreements arise over the measurements and the way of treating the patients, these issues are subjected to the personal experiences and emotional beliefs of the technicians and can turn into a moral challenge [15]. For example, an experienced emergency technician may determine that the patient's condition is not technically emergency, while another technician with less experience may consider it an emergency condition that requires urgent intervention.

Another issue related to professional communication that causes a moral challenge is the communication with staff in other units of the health system. Personnel from different units can be in disagreement over the patient's condition [6]. For example, according to emergency medical personnel, an emergency patient is diagnosed and requires follow-up at the hospital and is transferred to the hospital. Still, hospital staff refuses to admit and provide services to the patient.

\section{Safe driving}

EMS personnel drive at high speed to protect the lives of the injured patient while endangering their own safety. Some studies have been conducted on the impact of using sirens to increase the speed of ambulances and reduce the time to reach the scene [43]. This relates to situations of self-sacrifice: One of the dimensions of moral challenge that cannot be easily judged [42]. The use of sirens and alarms causes most ambulance accidents in the United States. The data show that $50 \%$ of ambulances' traffic accidents involved serious injury, and $1 \%$ resulted in death [30].

A recent epidemiological study of occupational accident deaths in prehospital service providers showed that out of 91 deaths, $74 \%$ were caused by ambulancerelated traffic accidents. It is, therefore, recommended to minimize the use of warning devices such as sirens and alarms [44]. Implementation of driving protocols without warning signs requires prioritizing medical emergency calls and dispatching ambulances according to the emergency and non-emergency conditions of patients through telephone triage of call center operators. On the other hand, research shows that the time required to complete the triage process before ambulance delivery is often considerable and time-consuming [45]. 


\section{Discussion and conclusion}

Prehospital care is a unique service and is characterized by factors that may lead to ethical challenges compared to other care services in different domains. At the same time, there are serious debates over ethical issues related to prehospital emergencies. The focus has been mainly on the ethical problems associated with doing and not doing cardiopulmonary resuscitation. The lack of a comprehensive and in-depth discussion of ethical issues has also been demonstrated in prehospital emergency instruction discussions. Most discussions lead to the recommendation and development of protocols that do not necessarily address ethical issues so that the best possible care for the patient is considered differently depending on the circumstances.

The values and norms that should guide prehospital emergency care include situations in which ethical decisions are often made within limited time and space. To solve these problems as best as possible, prehospital system technicians should consider possible options in each case against various conditions and opportunities, and do the best job of patient care and support by assessing the real situation.

\section{Ethical Considerations}

\section{Compliance with ethical guidelines}

All ethical principles were considered in this article.

\section{Funding}

This research did not receive any specific grant from funding agencies in the public, commercial, or not-forprofit sectors.

\section{Authors' contributions}

Study design: Ali Afshari, Mohammad Reza Yousefzadeh Chosari, Fatemeh Cheraghi; Data analysis, interpreting the results: Mehdi Beyrami Jam, Ali Afshari; Discussing the results and contributing in final manuscript: All authors.

\section{Conflict of interest}

The authors declared no conflict of interests.

\section{Acknowledgments}

Special thanks to the Director of the Disaster and Emergency Management Center of Hamedan province for helping the authors.

\section{References}

[1] French E, Casali GL. Ethics in emergency medical servicesWho cares? An exploratory analysis from Australia. EJBO. 2008; $13(2)$.

[2] Vryonides S, Papastavrou E, Charalambous A, Andreou P, Merkouris A. The ethical dimension of nursing care rationing: A thematic synthesis of qualitative studies. Nursing Ethics. 2015; 22(8):881-900. [DOI:10.1177/0969733014551377] [PMID]

[3] Karadag CO, Hakan AK. Ethical dilemmas in disaster medicine. Iranian Red Crescent Medical Journal. 2012; 14(10):602. [PMID] [PMCID]

[4] Butts JB. Adult health nursing ethics. Nursing Ethics. 2012:187.

[5] Sine DM, Sharpe VA. Ethics, risk, and patient $\square$ centered care: How collaboration between clinical ethicists and risk management leads to respectful patient care. Journal of Healthcare Risk Management. 2011; 31(1):32-7. [DOI:10.1002/ jhrm.20077] [PMID]

[6] Sandman L, Nordmark A. Ethical conflicts in prehospital emergency care. Nursing Ethics. 2006; 13(6):592-607. [DOI:10.1177/0969733006069694] [PMID]

[7] Erbay H. Some ethical issues in prehospital emergency medicine. Turkish Journal of Emergency Medicine. 2014; 14(4):193-8 [DOI:10.5505/1304.7361.2014.32656] [PMID] [PMCID]

[8] Essex B, Scott LB. Chronic stress andassociated coping strategies among volunteer ems personnel. Prehospital Emergency Care. 2008; 12(1):69-75. [DOI:10.1080/10903120701707955] [PMID]

[9] Petzäll K, Tällberg J, Lundin T, Suserud BO. Threats and violence in the Swedish pre-hospital emergency care. International Emergency Nursing. 2011; 19(1):5-11. [DOI:10.1016/j. ienj.2010.01.004] [PMID]

[10] Ripley E, Ramsey C, Prorock-Ernest A, Foco R, Luckett Jr S, Ornato JP. EMS providers and exception from informed consent research: benefits, ethics, and community consultation. Prehospital Emergency Care. 2012; 16(4):425-33. [DOI:10.3109/1090312 7.2012.702189] [PMID] [PMCID]

[11] Nordby H. Ethics in prehospital emergency medicine: An ethical dilemma in patient communication. Webmed Central Medical Ethics. 2013; 4(5):WMC004247.

[12] Bremer A, Herrera MJ, Axelsson C, Martí DB, Sandman L, Casali GL. Ethical values in emergency medical services: A pilot study. Nursing Ethics. 2015; 22(8):928-42. [DOI:10.1177/0969733014551597] [PMID]

[13] Özcan M, Akpinar A, Birgili F, Beydilli H. Ethical challenges in emergency medical services and ethical reasoning among emergency care providers. Acta Medica. 2014; 30:241 
[14] Lowthian JA, Jolley DJ, Curtis AJ, Currell A, Cameron PA, Stoelwinder JU, et al. The challenges of population ageing: Accelerating demand for emergency ambulance services by older patients, 1995-2015. Medical Journal of Australia. 2011; 194(11):574. [DOI:10.5694/j.1326-5377.2011.tb03107.x] [PMID]

[15] Gunnarsson BM, Stomberg MW. Factors influencing decision making among ambulance nurses in emergency care situations. International Emergency Nursing. 2009; 17(2):83-9. [DOI:10.1016/j.ienj.2008.10.004] [PMID]

[16] Dale J, Williams S, Foster T, Higgins J, Snooks H, Crouch R, et al. Safety of telephone consultation for "non-serious" emergency ambulance service patients. BMJ Quality \& Safety. 2004; 13(5):363-73. [DOI:10.1136/qshc.2003.008003] [PMCID]

[17] Schmidt T, Neely KW, Adams AL, Newgard CD, Wittwer L, Muhr M, et al. Is it possible to s afely triage callers to emsdispatch centers to alternative resources? Prehospital Emergency Care. 2003; 7(3):368-74. [DOI:10.1080/10903120390936590] [PMID]

[18] Silvestri S, Rothrock SG, Kennedy D, Ladde J, Bryant M, Pagane J. Can paramedics accurately identify patients who do not require emergency department care? Prehospital Emergency Care. 2002; 6(4):387-90. [DOI:10.1080/10903120290937987] [PMID]

[19] Stiell IG, Wells GA, DeMaio VJ, Spaite DW, Field BJ, Munkley DP, et al. Modifiable factors associated with improved cardiac arrest survival in a multicenter basic life support/ defibrillation system: Opals study phase I results. Annals of Emergency Medicine. 1999; 33(1):44-50. [DOI:10.1016/S01960644(99)70415-4]

[20] Bradley SM, Gabriel EE, Aufderheide TP, Barnes R, Christenson J, Davis DP, et al. Survival increases with CPR by emergency medical services before defibrillation of out-ofhospital ventricular fibrillation or ventricular tachycardia: Observations from the resuscitation outcomes consortium Resuscitation. 2010; 81(2):155-62. [DOI:10.1016/j.resuscitation.2009.10.026] [PMID] [PMCID]

[21] Atwood C, Eisenberg MS, Herlitz J, Rea TD. Incidence of EMS-treated out-of-hospital cardiac arrest in Europe. Resuscitation. 2005; 67(1):75-80. [DOI:10.1016/j.resuscitation.2005.03.021] [PMID]

[22] Lloyd-Jones D, Adams RJ, Brown TM, Carnethon M, Dai S, De Simone G, et al. Heart disease and stroke statistics-2010 update. Circulation. 2010; 121(7):e46-215. [DOI:10.1161/CIRCULATIONAHA.109.192666] [PMID]

[23] Millin MG, Khandker SR, Malki A. Termination of resuscitation of nontraumatic cardiopulmonary arrest: Resource document for the National Association of EMS Physicians position statement. Prehospital Emergency Care. 2011; 15(4):54754. [DOI:10.3109/10903127.2011.608872] [PMID]

[24] Brindis SL, Gausche-Hill M, Young KD, Putnam B. Universally poor outcomes of pediatric traumatic arrest: a prospective case series and review of the literature. Pediatric emergency care. 2011; 27(7):616-21. [DOI:10.1097/ PEC.0b013e31822255c9] [PMID]

[25] Lorenz HP, Steinmetz B, Lieberman J, Schecoter W, Macho JR. Emergency thoracotomy: survival correlates with physiologic status. The Journal of Trauma. 1992; 32(6):780-5. [DOI:10.1097/00005373-199206000-00020] [PMID]

[26] Hopson LR, Hirsh E, Delgado J, Domeier RM, McSwain NE, Krohmer J. Guidelines for withholding or termination of resuscitation in prehospital traumatic cardiopulmonary arrest: joint position statement of the National Association of EMS Physicians and the American college of surgeons committee on trauma. Journal of the American College of Surgeons. 2003; 196(1):106-12. [DOI:10.1016/S1072-7515(02)01668-X]

[27] Bailey ED, Wydro GC, Cone DC. Termination of resuscitation in the prehospital setting for adult patients suffering nontraumatic cardiac arrest. Prehospital Emergency Care. 2000; 4(2):190-5. [DOI:10.1080/10903120090941498] [PMID]

[28] Morrison LJ, Verbeek PR, Zhan C, Kiss A, Allan KS. Validation of a universal prehospital termination of resuscitation clinical prediction rule for advanced and basic life support providers. Resuscitation. 2009; 80(3):324-8. [DOI:10.1016/j.resuscitation.2008.11.014] [PMID]

[29] Beauchamp TL. The Belmont report. The Oxford textbook of clinical research ethics. Oxford: Oxford University Press; 2008.

[30] Becker TK, Gausche-Hill M, Aswegan AL, Baker EF, Bookman KJ, Bradley RN, et al. Ethical challenges in Emergency medical services: Controversies and recommendations. Prehospital and Disaster Medicine. 2013; 28(5):488-97. [DOI:10.1017/ S1049023X13008728] [PMID]

[31] Iserson KV. Bioethical dilemmas in emergency medicine and prehospital care. Health care ethics: Critical issues for the $21^{\text {st }}$ Century Sudbury: Jones and Bartlett Publishers. 2004:138-45.

[32] Pope TM. Legal briefing: Futile or non-beneficial treatment. The Journal of Clinical Ethics. 2011; 22(3):277-96.

[33] Sen G. Ethical decision-making by healthcare professionals during disasters: Izmir province case. World academy of science, engineering and technology. International Journal of Health and Medical Engineering. 2017; 4(7).

[34] Hanfling D, Altevogt BM, Viswanathan K, Gostin LO. Crisis standards of care: A systems framework for catastrophic disaster response. National Academies Press; 2012; 308(7):675-6. [DOI:10.1001/jama.2012.8171] [PMID]

[35] Nichol G, Thomas E, Callaway CW, Hedges J, Powell JL, Aufderheide TP, et al. Regional variation in out-of-hospital cardiac arrest incidence and outcome. JAMA. 2008; 300(12):142331. [DOI:10.1001/jama.300.12.1423] [PMID] [PMCID]

[36] Ihalainen $\square$ Tamlander N, Vähäniemi A, Löyttyniemi E, Suominen T, Välimäki M. Stigmatizing attitudes in nurses towards people with mental illness: A cross $\square$ sectional study in primary settings in Finland. Journal of Psychiatric and Mental Health Nursing. 2016; 23(6-7):427-37. [DOI:10.1111/ jpm.12319] [PMID]

[37] Ronzani TM, Higgins-Biddle J, Furtado EF. Stigmatization of alcohol and other drug users by primary care providers in Southeast Brazil. Social Science \& Medicine. 2009; 69(7):1080-4. [DOI:10.1016/j.socscimed.2009.07.026] [PMID]

[38] Erbay H, Alan S, Kadıŏglu S. A case study from the perspective of medical ethics: Refusal of treatment in an ambulance. Journal of Medical Ethics. 2010; 36(11):652-5. [DOI:10.1136/ jme.2010.035600] [PMID]

[39] Knapp BJ, Kerns BL, Riley I, Powers J. EMS-initiated refusal of transport: the current state of affairs. The Journal of Emergency Medicine. 2009; 36(2):157-61. [DOI:10.1016/j.jee mermed.2007.06.028] [PMID] 
[40] Grady C. Enduring and emerging challenges of informed consent. New England Journal of Medicine. 2015; 372(9):85562. [DOI:10.1056/NEJMra1411250] [PMID]

[41] Sirbaugh PE, Diekema DS. Consent for emergency medical services for children and adolescents. Pediatrics. 2011; 128(2):427-33. [DOI:10.1542/peds.2011-1166] [PMID]

[42] Larkin GL, Fowler RL. Essential ethics for EMS: Cardinal virtues and core principles. Emergency Medicine Clinics of North America. 2002; 20(4):887-911. [DOI:10.1016/S07338627(02)00034-2]

[43] Brown LH, Whitney CL, Hunt RC, Addario M, Hogue T. Do warning lights and sirens reduce ambulance response times? Prehospital Emergency Care. 2000; 4(1):70-4. [DOI:10.1080/10903120090941696] [PMID]

[44] Maguire BJ, Hunting KL, Smith GS, Levick NR. Occupational fatalities in emergency medical services: a hidden crisis. Annals of Emergency Medicine. 2002; 40(6):625-32. [DOI:10.1067/ mem.2002.128681] [PMID]

[45] Hinchey P, Myers B, Zalkin J, Lewis R, Garner Jr D. Low acuity EMS dispatch criteria can reliably identify patients without high-acuity illness or injury. Prehospital Emergency Care. 2007; 11(1):42-8. [DOI:10.1080/10903120601021366] [PMID] 
This Page Intentionally Left Blank 